\title{
Dental needs of intellectually disabled children attending six special educational facilities in Cape Town
}

\author{
T Roberts, ${ }^{1,2,4} \mathrm{BChD}, \mathrm{MChD}$; M Chetty, ${ }^{1,2,4} \mathrm{BChD}, \mathrm{MChD}$; F Kimmie-Dhansay, ${ }^{1} \mathrm{BChD}, \mathrm{MChD}$; \\ K Fieggen, ${ }^{3}$ MB ChB, FCPaed (SA), Cert Med Genet; L X G Stephen, ${ }^{1,2}$ BChD, PhD \\ ${ }^{1}$ Diagnostics Cluster, Faculty of Dentistry, University of the Western Cape, Cape Town, South Africa \\ ${ }^{2}$ University of the Western Cape/University of Cape Town Special Dental Genetic Clinic; and Red Cross War Memorial Children's Hospital, Cape \\ Town, South Africa \\ ${ }^{3}$ Division of Human Genetics, Department of Pathology, Faculty of Health Sciences, University of Cape Town, South Africa \\ ${ }^{4}$ Division of Human Genetics, Department of Pathology, Faculty of Health Sciences, University of Cape Town, South Africa. Currently postgraduate/ \\ PhD student
}

Corresponding author: T Roberts (tinasharonroberts@gmail.com)

\begin{abstract}
Objective. To assess the dental needs of a group of children with intellectual disability (ID) attending six special educational facilities in Cape Town, South Africa.

Methods. This was a cross-sectional study based on a convenience sampling method. One hundred and fifty-seven children with ID attending six special educational facilities in Cape Town were included in the survey. Five schools were exclusively funded by the State and one school received additional private financial support. The oral examinations complied with guidelines drafted by Special Olympics Special Smiles programme and the Centers for Disease Control, USA.

Results. The most common dental disorders requiring management were gingival disease (69\%) and untreated dental caries (68\%). Almost 50\% of the children had missing teeth. Twenty-nine percent needed orthodontic correction of malocclusion and $7 \%$ had structural abnormalities of their teeth that required either aesthetic or functional intervention. Fillings were evident in only $8 \%$ of the children. Females required more dental treatment than males. The dental needs of children with ID increased with age. There were no significant differences in the dental needs of children attending State-funded schools and those attending the single school that received additional financial assistance.

Conclusion. The frequency of unmet dental needs of children with ID attending special educational facilities in Cape Town was high and the dental care available to them was minimal. The study highlights the need for improved dental services to ensure that optimal oral health is accessible to children with ID attending special educational facilities in Cape Town.
\end{abstract}

S Afr Med J 2016;106(6):S94-S97. DOI:10.7196/SAMJ.2016.v106i6.11006

Intellectual disability (ID) is a common and important problem that has many social and medical implications. ${ }^{[1]}$ Disorders of orodental structures often arise as a result of oral and dental abnormalities co-occurring with ID. ${ }^{[2]}$

There are various definitions of ID and its diagnosis primarily depends on the country and the context where it is being reported. Population surveys and information collected from school referrals are the primary sources of prevalence data. For these reasons, the documented prevalence of ID varies between studies from the same country as well as between different countries. ${ }^{[3-5]}$

Prevalence data for ID for Africa are available from the literature. In Mozambique, the prevalence is $1.9 \%$ in rural areas v. $1.3 \%$ in urban areas; ${ }^{[3]}$ in Ethiopia the prevalence is $10.3 \%{ }^{[4]}$ and in Nigeria $6.2 \%{ }^{[5]}$ The most recent estimated prevalence of ID for children aged 5 years and older in SA is 3.2\% for mild disability and $1 \%$ for severe impairment. In the latter survey, ID was diagnosed when moderate to severe limitations were present in at least three areas of recall or concentration, communication and selfcare. ${ }^{[6]}$ Selfcare includes daily routines such as bathing, brushing hair and brushing teeth. Gingival disease and dental caries are two significant results of inadequate oral care. ${ }^{[7]}$

When children with ID seek dental treatment, the management is either curative or restorative, as opposed to preventive. Furthermore, the majority of the procedures require general anaesthesia, sedation or specialised intervention. In addition, the demand for general anaesthesia for routine dentistry in the Western Cape is high. ${ }^{[8]}$ It is therefore imperative to determine the prevalence of dental needs among children with ID in order to promote strategies for oral hygiene and prevention among caregivers and affected children.

The aim of the investigation was to assess the unmet dental needs, to provide urgent dental treatment and to explore arrangements for future dental management. The orodental problems and complications encountered during the investigation are documented in this article.

\section{Objectives}

To identify the dental needs of a group of children with ID attending six special educational facilities in Cape Town, South Africa (SA), specifically:

- To determine the following clinical parameters of the participants:

- the presence of dental caries and gingival disease following the guidelines formulated by Special Olympics Special Smiles (SOSS) and the Centers for Disease Control (CDC), USA

- the prevalence rates for missing and filled teeth, malocclusion and structural tooth abnormalities

- To compare the frequency of individual clinical parameters between specific age groups and genders

- To determine the effect of additional financial support of an institution on dental care.

\section{Methods}

The study was cross-sectional in design. 


\section{Eligibility}

Children with ID attending any one of the six schools on the day of examination, whose parents gave consent, were included in the study. Those whose parents did not consent to evaluation were excluded.

\section{Study population}

The study population consisted of children with ID between the ages of 3 and 19 years, attending six special educational facilities in Cape Town. Parental consent and learner's assent were sought in every instance and 10 children who refused dental assessment were excluded from the investigation. The study was reviewed and approved by the University of Cape Town (UCT) Faculty of Health Sciences Ethics Committee (Ref. 021/2010) and was conducted according to the Code of Ethics outlined in the Belmont Report. ${ }^{[9]}$

\section{Study sample}

The six schools were selected for logistical reasons and because all new learners received thorough diagnostic assessment and psychological screening before entering the facility. Admission to a particular special educational facility was dependent on the proximity of parents' residential home to the school. Financing of the schools was through the State (Department of Education, Western Cape Government). One of the schools received additional funding from a private charitable source.

Nursing care was available at each of the schools, but none had dedicated dental facilities. The parents occasionally arranged tooth extractions and other minor dental procedures, but many children lacked care of this type.

A clinical team from the Division of Human Genetics, UCT, regularly visits the schools for diagnostic evaluation of the learners to ascertain the possibility of a genetic cause of the children's disability. The team was accompanied by a qualified dentist (TR) in order to carry out dental assessments.

A resident member of staff at each school selected the children with ID for genetic evaluation. All children examined by the genetics team were referred for dental appraisal; hence, the sample size was determined by convenience sampling. Thereafter, the dentist undertook orodental assessment and arranged for any necessary dental treatment. The Faculty of Dentistry, University of the Western Cape provided sophisticated dental management if this was necessary. Members of the team documented clinical, medical and dental case histories and performed the clinical examinations. The dental information was recorded on a specifically designed data collection sheet.
The data were stored using Microsoft Excel. A password secured the laptop holding the information, ensuring the confidentiality of participants' information, using a designated code for each participant.

Dental assessments were undertaken using natural light and a mouth mirror. Gloves, masks and autoclaved instruments formed the basis for strict infection control practices. ${ }^{[10]}$

\section{Measurements}

Oral screening was undertaken on all assenting children, following a protocol formulated by the SOSS and the CDC. ${ }^{[11]}$ Demographic (independent) variables included age, gender, disability, school, and type of school according to funding. Categorical dependent data included decayed, filled and missing teeth; gingival disease (involving three or more teeth); malocclusion; and structural abnormalities of teeth.

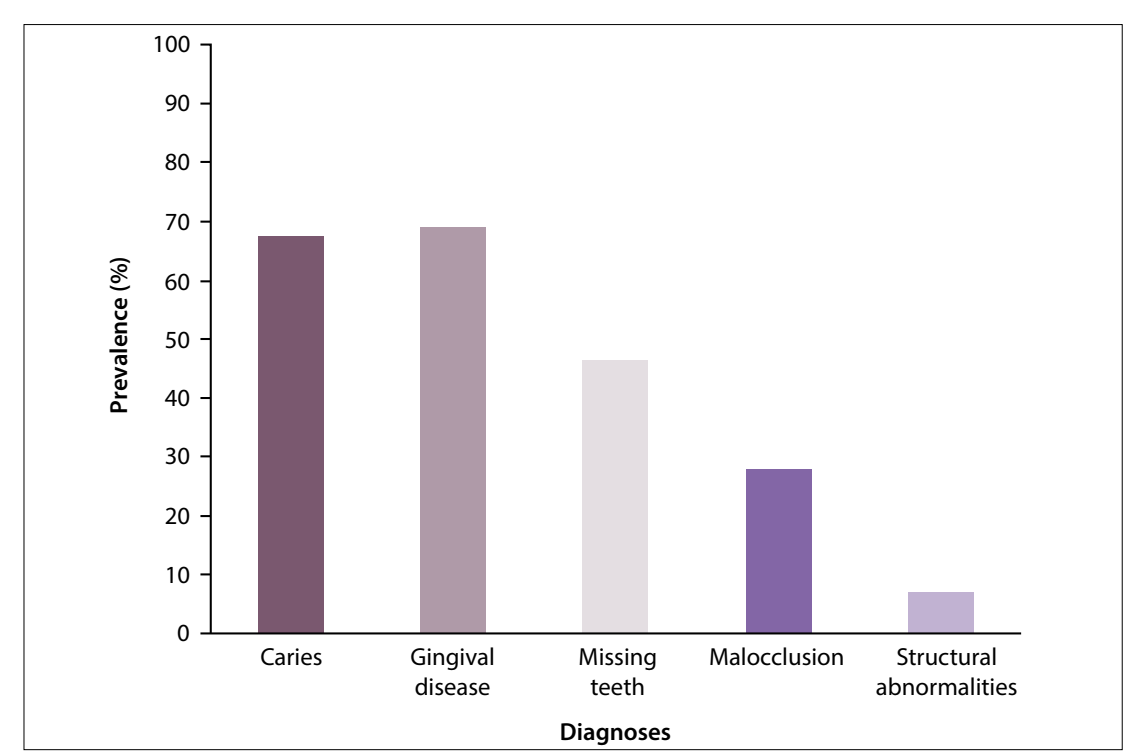

Fig. 1. Prevalence of caries, gingival disease, missing teeth, malocclusion and structural abnormalities.

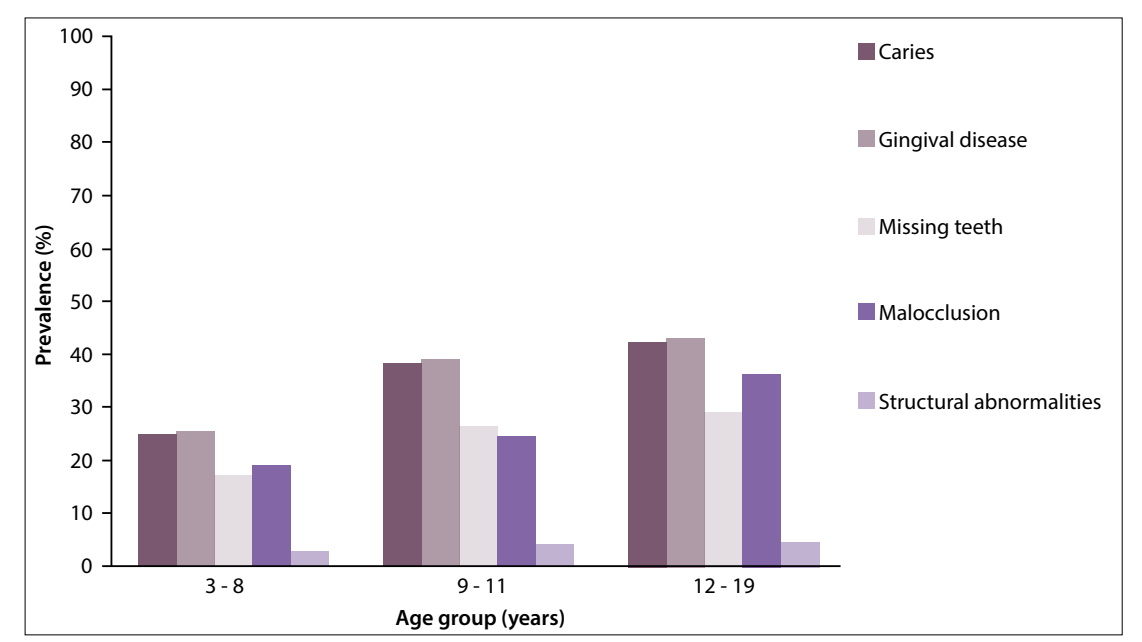

Fig. 2. Prevalence of caries, gingival disease, missing teeth, malocclusion and structural abnormalities

by age group.

Changes in the contour and texture of the gingiva in at least three or more teeth were classified as gingival disease. Caries was defined as at least one area of recognisable cavitation $>0.5 \mathrm{~mm}$ in diameter in either the primary or the permanent dentition. ${ }^{[1]}$ Missing teeth were documented as teeth absent at the time of examination.

\section{Statistical analysis}

The collected data were entered into a Microsoft Excel spreadsheet. A professional statistician analysed the findings using STATA Release 12 (Statacorp, USA). Categorical data were evaluated using a $\chi^{2}$ test and if assumptions were not satisfied, Fisher's exact test was preferred. A $p$-value $<0.05$ rendered statistical significance.

\section{Results}

Oral screening was undertaken in 157 children attending special educational facilities 
in Cape Town, of whom 105 (67\%) were male and $52(33 \%)$ were female. The average age was 10.1 years (SD 3.42). There was no statistically significant difference in gender distribution across the different age groups $(p=0.614)$.

Caries prevalence was $67.5 \%, n=106$ (Fig. 1). Caries frequency increased as the age group of the sample increased. However, the escalation was not statistically significant $(p=0.167)$ and was similar between males and females $(p=0.225)$ (Figs 2 and 3 ).

Sixty-nine percent $(n=108)$ of the participants had evidence of gingival disease. This variable increased with age and occurred in only $25 \%(n=24)$ of participants in the 3 8 years age group. Although this figure was much lower than in the older age groups, it was not statistically significant $(p=0.259$ ).

Missing teeth were documented in $46.5 \%(n=73)$ of the participants (Fig. 1). Participants in the 12 - 19-years age group presented with the highest prevalence of missing teeth $(29.3 \%, n=20)$. The prevalence of missing teeth was statistically significant between age groups ( $p=0.009$; Fig. 2 ).

Almost $30 \%(n=44)$ of participants presented with malocclusion (Fig. 1). The presence of malocclusion was statistically different between the different age groups $(p=0.041)$. Malocclusion occurred in $18.9 \%$ $(n=7)$ of children aged 3 - 8 years compared with $24.6 \%(n=14)$ and $36.5 \%(n=23)$ in those aged $9-11$ years and $12-19$ years, respectively. There was no statistically significant difference in the prevalence of malocclusion between males $(26.7 \%, n=28)$ and females (30.8\%, $n=16)$ ( $p=0.797$; Fig. 3 ).

\section{Discussion}

This is the first description of the oral health needs of children with ID attending special educational facilities in Cape Town and in SA. The main purpose of this investigation was to identify the dental needs in a group of children with ID at six special educational facilities in Cape Town. These children had ID of mild to moderate severity and for this reason different regimens of orodental care were necessary. The most frequent dental needs among these children were the treatment of gingival disease and dental caries, both of which are avoidable disorders. The need for routine dental intervention and orthodontic management of malocclusion was highest in the 12 - 19-year age group. The findings of the investigation show that the prevalence of caries, gingival disease, missing teeth and malocclusion increases with age in this population group. However, only the frequency of missing teeth and the presence of malocclusion showed statistical significance.

Children attending the school that receives additional funding required more dental intervention than did those at schools relying solely on financial support from the government. This may indicate that more emphasis could be placed on managing aspects of ID not related to dental needs.

The prevalence of gingival disease in the children in this study (69\%) was similar to that reported in certain lower-middleincome economies but lower than reported in some high income and other lowermiddle-income economies. ${ }^{[12-14]}$ Untreated gingival disease may result in chronic inflammation-induced periodontitis, a condition commonly affecting certain groups of individuals with ID such as those with Down syndrome.

Sixty-seven percent of the children in the study had one or more carious lesions. The figure is higher than that reported in the

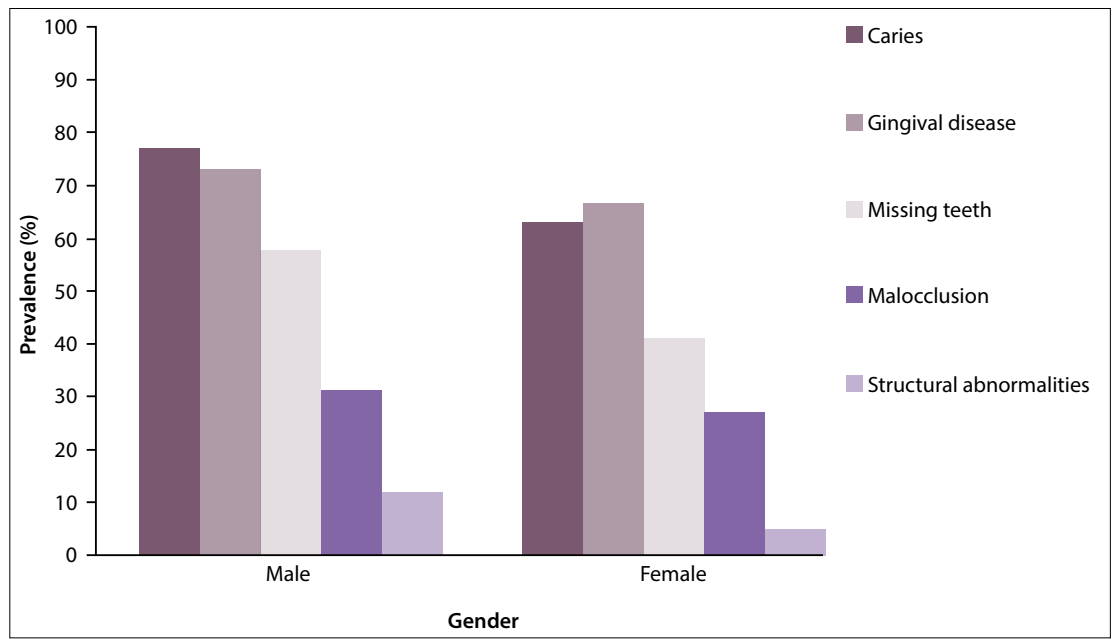

Fig. 3. Prevalence of caries, gingival disease, missing teeth, malocclusion, structural abnormalities by gender. general SA population of similar age but similar to the report on children with ID in Gauteng Province, SA. ${ }^{[15]}$ It is also similar to that reported in some lower-middleincome economies in children with ID, with the exception of Nigeria, where caries was uncommon. ${ }^{[14,16]}$

Children with ID are at a higher risk of developing dental caries than the general population. ${ }^{[17]}$ The type of school they attend, the diet they follow and the severity of their ID are factors that may contribute to the burden of poor oral health. ${ }^{[18]}$

Dental caries has a significant influence on the wellbeing of children with ID. Extensive carious lesions may hinder speech and mastication, resulting in malnutrition and bowel irregularities. ${ }^{[19]}$ The oral cavity is a source of numerous pathogens, and children with comorbid disorders such as cardiac defects will benefit from good oral hygiene by elimination of unnecessary sources of bacteria.

The high percentage of missing teeth in this population is attributable to the natural course of tooth eruption and exfoliation, and the high frequency of dental caries.

Thirty percent of the children examined had malocclusion. This is lower than reported elsewhere. ${ }^{[16,20]}$ The term 'malocclusion' includes cross-bites, open bites and overbites; these are either localised or generalised. Although malocclusion occurs in the general population, there appears to be a higher prevalence in people with ID. ${ }^{[20]}$

Management of both malocclusion in children with ID depends on the type and severity of the disability, as well as the nature of the malocclusion. Orthodontic treatment to correct malocclusion in children with ID is similar to that in unaffected children, and ID should not be considered as an obstacle to receiving optimal care. In general, children with orthodontic appliances need to maintain exceptional oral hygiene. When children are affected by ID, caregivers may need to play a greater role in order to maintain satisfactory oral hygiene. Despite these considerations, none of the survey children affected by malocclusion received either interceptive or curative intervention. Access to such specialised management was limited, mainly by the high cost of management in private practice and long waiting lists at specialised hospitals.

\section{Conclusion}

The study highlights the need for improved dental services to ensure that optimal oral health is accessible to children with ID attending special educational facilities in Cape Town. The adverse dental outcomes 
evident in the older group of children indicate that the treatment needs among the children in this population are inadequately met. In addition, they suggest that poor dental care does not solely depend on the financial support of an institution.

Regardless of the cause or severity of ID, there is a significant lack of access to good quality dental and medical care worldwide. The dental care needs of those with ID often remain unmet due to a shortage of enthusiastic and specially trained dental professionals to provide dental treatment, and limited dental resources for the population as a whole.

There were potential limitations to the study. These include that participants' medical records were not always available during the screening sessions, and that the dietary status of the participants was not taken into account.

\section{References}

1. May ME, Kennedy $\mathrm{CH}$. Health and problem behavior among people with intellectual disabilities. Behav Anal Pract 2010;3(2):4-12.

2. Solanki J, Khetan J, Gupta S, Tomar D, Singh M. Oral rehabilitation and management of mentally retarded. J Clin Diagnostic Res 2015;9(1):ZE01-ZE06. DOI:10.7860/JCDR/2015/11077.5415

3. Patel V, Simbine APF, Soares IC, Weiss HA, Wheeler E. Prevalence of severe mental and neurological disorders in Mozambique: A population-based survey Lancet 2007; 370(9592):1055-1060. DOI:10.1016/s0140-6736(07)61479-2

4. Fitaw Y, Boersma JMF. Prevalence and impact of disability in north-western Ethiopia. Disabil Rehabil 2006;28(15):949-953. DOI:10.1080/09638280500404552

5. Ologe FE, Akande TM. Pattern of disabilities in a residential school for the handicapped in Ilorin, Nigeria. Niger Postgrad Med J 2003;10(4):208-210.

6. Statistics South Africa. Census 2011. Profile of persons with disabilities in South Africa. Pretoria: SSA, 2014. http://www.statssa.gov.za/publications/Report-03-01-59/Report-03-01-592011.pdf (accessed 14 April 2015).
7. World Health Organization. Oral Health. Geneva: WHO, 2012. http://www.who.int/mediacentre/ factsheets/fs318/en/index.html (accessed 14 April 2015).

8. Peerbhay F, Barrie RB. The burden of early childhood caries in the Western Cape Public Service in relation to dental general anaesthesia: Implications for prevention. SADJ 2012;67(1):14-16,18-19.

9. The National Commission for the Protection of Human Subjects of Biomedical and Behavioral 9. The National Commission for the Protection of Human Subjects of Biomedical and Behavioral
Research. The Belmont report: Ethical principles and guidelines for the protection of human subjects of research. Bethesda: NIH OHSR, 1979:1-10.

10. Centers for Disease Control. CDC infection control for dentists. Morbidity and Mortality Weekly Report. Atlanta: CDC, 2001. http://www.cdc.gov/mmwr/preview/mmwrhtml/00033634.htm (accessed 14 April 2015).

11. White JA, Beltran ED. Training Manual for Standardized Oral Health Screening. Atlanta: Centers for Disease Control, 2004. http://media.specialolympics.org/soi/files/healthy-athletes/HA_SS Training+Manual.pdf (accessed 14 April 2015).

12. Kumar S, Sharma J, Duraiswamy P, Kulkarni S. Determinants for oral hygiene and periodontal status among mentally disabled children and adolescents. J Indian Soc Pedod Prev Dent 2009;27(3):151-157. DOI: $10.4103 / 0970-4388.57095$

13. Anders PL, Davis EL. Oral health of patients with intellectual disabilities: A systematic review. Spec Care Dent 2010;30(3):110-117. DOI:10.1111/j.1754-4505.2010.00136x

14. Gardens SJ, Krishna M, Vellappally S, et al. Oral health survey of 6-12-year-old children with disabilities attending special schools in Chennai, India. Int J Paediatr Dent 2014;24(6):424-433. DOI:10.1111/ipd.12088

15. Nqcobo CB, Yengopal V, Rudolph MJ, Thekiso M, Joosab Z. Dental caries prevalence of children attending special needs schools in Johannesburg, Gauteng, South Africa. SADJ 2012;67(7):308 313.

16. Oredugba FA, Akindayomi Y. Oral health status and treatment needs of children and young adults attending a day centre for individuals with special health care needs. BMC Oral Health 2008;22(8):30 DOI: $10.1186 / 1472-6831-8-30$

17. Liu Z, Yu D, Luo W, et al. Impact of oral health behaviors on dental caries in children with intellectua disabilities in Guangzhou, China. Int J Environ Res Public Health 2014;11(10):11015-11027. DOI:10.3390/ijerph111011015

18. Alaki SM, Bakry NS. Dental pain in children with intellectual disabilities: Caregivers' perspective. Int J Dent 2012;2012:701608. DOI:10.1155/2012/701608

19. Abanto J, Paiva SM, Raggio DP, Celiberti P, Aldrigui JM, Bönecker M. The impact of dental caries and trauma in children on family quality of life. Community Dent Oral Epidemiol 2012;40(4):323-331. DOI:10.1111/j.1600-0528.2012.00672

20. Purohit BM, Acharya S, Bhat M. Oral health status and treatment needs of children attending special schools in South India: A comparative study. Spec Care Dent 2010;30(6):235-241. DOI:10.1111/j.17544505 $2010.00160 . x$ 